Revista Destaques Acadêmicos, Lajeado, v. 11, n. 4, 2019. ISSN 2176-3070

DOI: http://dx.doi.org/10.22410/issn.2176-3070.v11i4a2019.2400

http://www.univates.br/revistas

\title{
METODOLOGIAS ATIVAS NO ENSINO DE ALGORITMOS E PROGRAMAÇÃO: UM RELATO DE APLICAÇÃO DA METODOLOGIA PEER INSTRUCTION
}

\author{
Mouriac Halen Diemer ${ }^{1}$, Magda Bercht ${ }^{2}$, Alberto Bastos do Canto Filho ${ }^{3}$, \\ Maria Claudete Schorr ${ }^{4}$
}

Resumo: As dificuldades com o aprendizado de algoritmos são uma das razões para os altos índices de desistência nos cursos de computação e informática, motivando a adoção de novas estratégias de ensino. Este artigo apresenta os resultados alcançados com a aplicação da metodologia ativa de aprendizagem Peer Instruction no ensino de algoritmos em turmas de graduação. Foram comparadas as médias históricas dos estudantes expostos a aulas convencionais com a média de quatro turmas de alunos que experienciaram a metodologia. A análise dos resultados foi realizada qualitativamente com o apoio de ferramentas quantitativas. O presente trabalho mostrou resultados positivos, indicando a consecução dos objetivos pedagógicos e evidenciando que o uso da metodologia Peer Instruction corroborou para a melhoria do desempenho dos alunos.

Palavras-chave: Peer Instruction; Ensino de Programação; Trajetórias de Aprendizagem.

1 Doutorando do Programa de Pós-Graduação em Informática na Educação (PGIE) da Universidade Federal do Rio Grande do Sul (UFRGS). Professor da Universidade do Vale do Taquari (UNIVATES). Graduado em Informática pela Universidade do Vale do Rio dos Sinos (UNISINOS) e mestre em Computação pela Universidade Federal do Rio Grande do Sul (UFRGS).

2 Professora e orientadora no Programa de Pós-Graduação em Informática na Educação (PGIE) da Universidade Federal do Rio Grande do Sul (UFRGS). Doutora em Computação pela Universidade Federal do Rio Grande do Sul (UFRGS).

3 Professor e orientador no Programa de Pós-Graduação em Informática na Educação (PGIE) da Universidade Federal do Rio Grande do Sul (UFRGS). Doutor em Informática na Educação pela Universidade Federal do Rio Grande do Sul (UFRGS).

4 Doutoranda do Programa de Pós-Graduação em Informática na Educação (PGIE) da Universidade Federal do Rio Grande do Sul (UFRGS). Professora da Universidade do Vale do Taquari (UNIVATES). Licenciada em Computação pela Universidade de Santa Cruz do Sul (UNISC) e mestre em Ensino de Ciências Exatas pela Universidade do Vale do Taquari (UNIVATES). 


\section{INTRODUÇÃO}

Conforme as Diretrizes Curriculares Nacionais para computação ${ }^{5}$ (BRASIL, 2016), os bacharelados desta área devem possuir uma base sólida de conteúdos relacionados ao raciocínio lógico e à programação de computadores. Para os estudantes dos cursos de computação, este processo é um dos pilares da formação. A base de conhecimentos consolidados pelos discentes nos semestre iniciais da formação irá influenciar no desempenho deles ao longo da trajetória universitária (NOSCHANG; PELZ; RAABE, 2014).

Assim, um dos desafios dos coordenadores de curso, professores e instituições de ensino que ofertam cursos de computação é melhorar os índices de aprovação nas disciplinas responsáveis pelo ensino de programação, tanto pela importância que tem esse conteúdo na formação desses profissionais, quanto pela manutenção dos estudantes no currículo (VIEIRA; DE LIMA JUNIOR; DE PAULA VIEIRA, 2015). Em relação ao aprendizado de algoritmos, por ser normalmente abordado nas primeiras disciplinas dos currículos da área de computação e informática e por ser o primeiro contato dos estudantes com a lógica de programação $0^{6}$ :

[...] observa-se de forma bastante significativa que ela [referindo-se ao aprendizado de algoritmos] constitui grande "divisor de águas" nos cursos de Computação, pois causa grande impacto no primeiro ano de formação, evidenciando de forma indireta, para quem não consegue compreender o assunto, uma grande barreira que impede a progressão do discente para outros períodos e não somente isso, o entendimento de disciplinas de programação que também fazem parte do currículo (VIEIRA; DE LIMA JUNIOR; DE PAULA VIEIRA, 2015, p.6).

O Senso da Educação Superior (BRASIL, 2018), mostra que a desistência no ensino superior brasileiro é grande em todas as áreas, formando menos da metade dos ingressantes, mas nos cursos de computação em média apenas $1 / 4$ dos ingressantes chegam ao final do percurso. De acordo com estudos realizados por Hoed (2016, p. 82) "a evasão em meio aos reprovados em disciplinas de algoritmos passa a ser maior já a partir do segundo período, sendo uma evidência de que o mau rendimento em disciplinas de lógica computacional

5 As DCN - Diretrizes Curriculares Nacionais para os cursos da área de computação propõe e normatizam quatro diferentes terminalidades para formação de profissionais nessa área: Ciência da Computação, Engenharia de Software, Engenharia de Computação e Sistemas de Informação.

6 Alguns currículos possuem uma disciplina inicial que aborda exclusivamente lógica formal ou proposicional. Os algoritmos podem também ser representados graficamente, usando pseudocódigo ou codificados em uma linguagem de programação. No contexto deste trabalho isso é compreendido como uma área de ensino. 
motiva a evasão". Os baixos índices de aprovação e os altos índices de abandono do curso são reflexos decorrentes das dificuldades enfrentadas pelos estudantes (BARROS; SANTOS, 2018; GIRAFFA; MÜLLER, 2017). Vencer as dificuldades iniciais de aprendizagem de lógica de programação reduz a probabilidade de evasão, segundo estudo realizado por Abu-Oda e El-Halees (2015).

Neste sentido, cresce em importância, entre os docentes que atuam ensinando programação, o desejo de aplicar novas estratégias pedagógicas, motivados pelo perfil dos ingressantes e dada a relevância deste conteúdo curricular na formação e na permanência dos estudantes (BARCELOS, 2013; BULCÃO; CAMPOS NETO; MOREIRA, 2017; CASTANHA; DE CASTRO, 2010; VASCONCELOS et al., 2019).

Considerando que cada estudante percorre uma trajetória de aprendizagem própria com pontos de partida dispersos, pois possuem conhecimentos prévios diferentes, o presente estudo se propôs a avaliar a aplicação da metodologia ativa Peer Instruction no aprendizado de algoritmos, tendo a intencionalidade de responder à seguinte questão: a metodologia Peer Instruction corrobora com o aprendizado de lógica de programação, auxiliando os discentes a compreenderem o processo de construção de programas de computadores?

\section{$1.2 \mathrm{O}$ ensino de algoritmos}

As competências requeridas para programar, particularmente as relacionadas ao uso adequado do raciocínio lógico e matemático para resolução de problemas, configuram-se como um dos maiores desafios que enfrentam os calouros $^{7}$ (BARROS; SANTOS, 2018). As barreiras que dificultam o aprendizado de algoritmos têm diferentes origens e naturezas, compreendendo questões de ordem didática, afetiva e cognitiva (RAABE; SILVA, 2005).

Um agravante para vencer as barreiras impostas pelas dificuldades inerentes à lógica de programação são as lacunas da formação básica. Uma parcela importante dos estudantes que iniciam um curso superior, apresentam deficiências, geralmente quanto à expressão em língua vernácula, interpretação de textos e, especialmente, de matemática (LOBO 2012 apud ALVES; GUIMARÃES; PIMENTA, 2018; BARCELOS; SILVEIRA，2012; BULCÃO; CAMPOS NETO; MOREIRA, 2017).

Independente de qual for a origem das dificuldades é consenso entre os docentes que atuam com algoritmos e programação de que elas existem e que estratégias de ensino diferentes precisam ser experimentadas (BULCÃO; CAMPOS NETO; MOREIRA, 2017), visando a maximizar o aprendizado e motivar os estudantes a permanecerem no curso. Neste sentido, o uso de metodologias ativas que promovem a centralidade do estudante e priorizam a

7 Estudantes do Ensino Superior. 
aprendizagem colaborativa por meio de trabalhos em grupo, vem sendo objeto de estudo e impulsionando mudanças curriculares e pedagógicas (BERBEL, 2011; BONDIOLI; VIANNA; SALGADO, 2019; BULCÃO; CAMPOS NETO; MOREIRA, 2017; VASCONCELOS et al., 2019).

\subsection{As metodologias de aprendizagem ativas}

Durante anos o ensino foi orientado para reprodução (quase mecânica) dos conteúdos apresentados pelo professor. O modelo transmissivo tem sido a forma predominante de instrução, desde que as primeiras universidades foram fundadas na Europa Ocidental há mais de 900 anos, onde os alunos recebem passivamente informações do professor (FREEMAN et al., 2014). A condição que põe o docente em um palco expondo conteúdos e esperando respostas corretas dos alunos não cabe mais. Os estudantes precisam desenvolver competências científicas e tecnológicas como argumentação, comunicação e validação em situações que de fato os façam refletir sobre os conceitos e suas aplicações práticas, ou seja, que lhes permitam aprender (VASCONCELOS et al., 2019).

O uso de novos recursos pedagógicos ou novas metodologias vem sendo discutido fortemente nos últimos anos em diferentes instâncias e fóruns. A Declaração de Santo Domingo (A Ciência para o Século XXI), por exemplo, afirma que a sociedade do conhecimento "implica o aumento das capacidades tecnológicas, combinando metodologias tradicionais e modernas, que estimulem a criação científica e tornem viável o desenvolvimento humano sustentável" (UNESCO, 1999 apud BERNHEIM; CHAUÍ, 2008, p. 16).

Neste sentido as metodologias de ensino ativas constituem-se como ferramentas que podem auxiliar o estudante em sua trajetória de aprendizagem que une as pontas entre os conhecimentos prévios e os objetivos propostos para uma determinada unidade de estudos, pois exploram a "problematização como estratégia de ensino e aprendizagem, com o objetivo de alcançar e motivar o discente, pois diante do problema, ele se detém, examina, reflete, relaciona a sua história e passa a ressignificar suas descobertas" (MITRE et al., 2008, p. 2135). A aprendizagem ativa envolve os alunos por meio de atividades ou discussão em sala de aula, ocorre quando o aluno interage com o assunto, enfatizando o pensamento de ordem superior (ouvindo, falando, escutando, perguntando, discutindo, fazendo e ensinando) e geralmente envolvendo trabalho em grupo (BARBOSA; DE MOURA, 2013; BERBEL, 2011; FREEMAN et al., 2014; GIRAFFA; MÜLLER, 2017; VASCONCELOS et al., 2019).

\subsubsection{A aprendizagem por pares}

A aprendizagem por pares caracteriza-se como uma metodologia onde a troca de experiências e conhecimentos entre os educandos é estimulada pelo docente. Diferentes estratégias podem ser exploradas para fomentar a 
interação, bem como a discussão e o debate acerca de determinado conceito ou assunto. Uma das metodologias que orientam o trabalho docente na promoção do aprendizado entre pares é o Peer Instruction.

A metodologia Peer Instruction foi proposta formalmente por Eric Mazur, professor de física na Universidade de Harvard, em 1997 (MAZUR; HILBORN, 1997). A metodologia preconiza que os estudantes tenham contato prévio com o conteúdo (antes da aula) e depois, durante a aula, o docente faz explanações curtas complementares ao estudo já realizado. Após a explanação o professor submete os discentes a um problema real, conforme é demonstrado na Figura 01, questionando-os ou desafiando-os a resolver um caso prático onde aquele conteúdo se faz necessário. Valendo-se de algum mecanismo de questionamento, o professor obtém feedback sobre o grau de compreensão da explanação realizada. Se o índice de êxito é muito baixo (inferior a 30\%) o professor deve complementar a sua explicação e refazer o processo de questionamento. Quando o índice de acertos é superior a 70\% a metodologia preconiza que houve compreensão dos conceitos pela maioria dos estudantes e que é possível seguir em frente. A troca de experiência entre os pares ocorre justamente quando o índice de acertos fica entre 30\% e 70\%, indicando que uma parcela dos estudantes já compreendeu o conteúdo e outra parcela ainda carece de auxílio e mais tempo. Os alunos, então, devem formar grupos de estudo com colegas que têm respostas divergentes, discutir o assunto, argumentar e defender seus pontos de vista e chegar a uma resposta comum. Este é o ponto máximo da metodologia, onde há intensa reflexão e postura ativa dos estudantes no processo de aprendizagem. Depois deste período de discussão um novo feedback é colhido pelo professor e conforme o índice de acertos o professor decide complementar ou não com novo momento de explanação ou apenas com uma reflexão de consolidação do tema. 
Figura 01 - Esquema de funcionamento da metodologia Peer Instruction

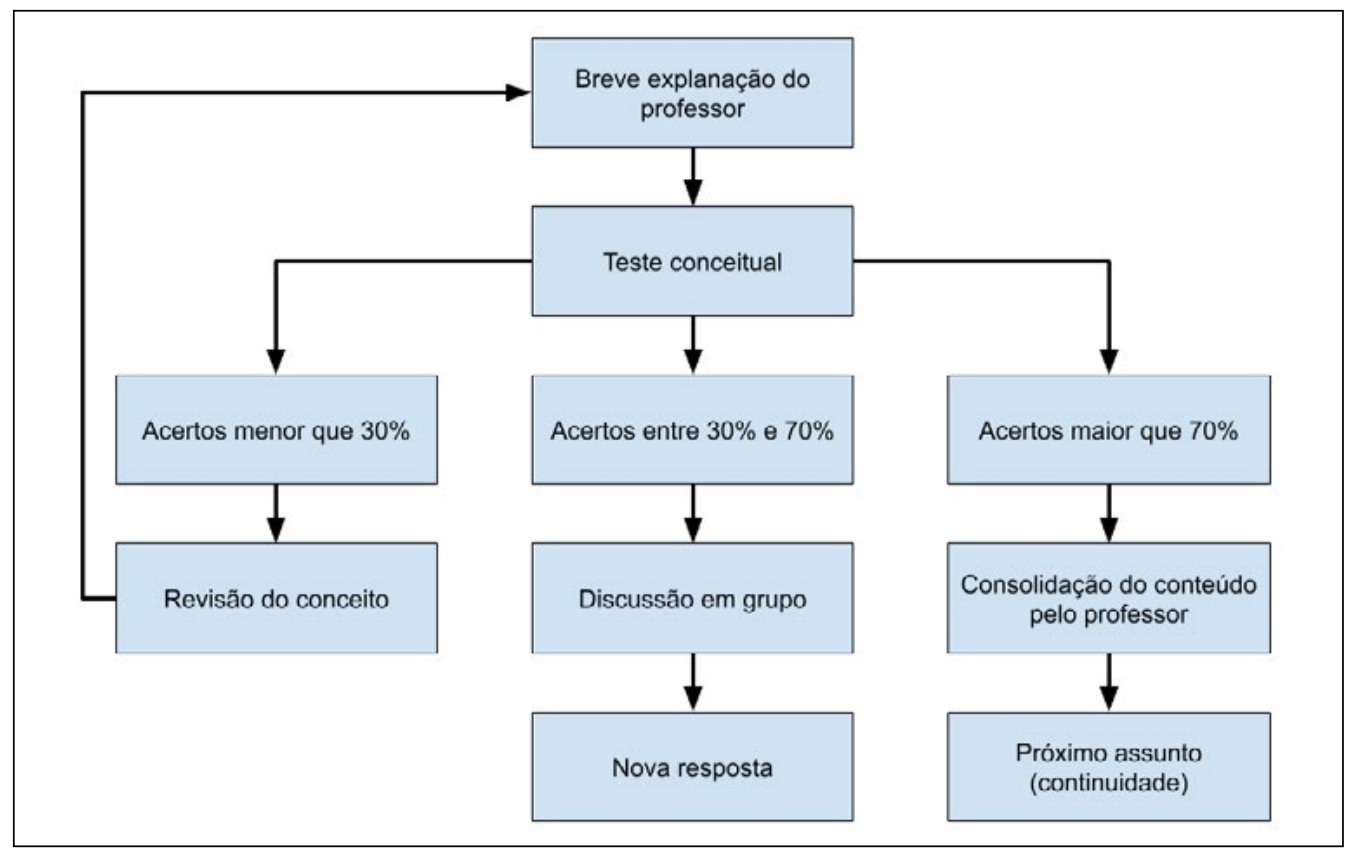

Fonte: adaptado de Mazur (1997)

A metodologia Peer Instruction promove, portanto, a colaboração e a interação entre os estudantes. Segundo Henri (2002 apud TORRES, 2002, p. 45), a interação é uma das características mais importantes da comunicação e é um dos fatores determinantes da aprendizagem. A colaboração, outrossim, pode ser vista como "qualquer atividade na qual duas ou mais pessoas trabalham juntas para criar significado, explorar um tópico ou melhorar habilidades" (HARISIM, 1995 apud TORRES, 2002, p. 41).

\subsection{Objetivo}

Tomando como premissa que os conhecimentos prévios e as experiências pregressas que serviram de base para construção dos esquemas mentais de raciocínio dos indivíduos de uma turma são diferentes, entende-se então que os pontos de partida são singulares e provavelmente dispersos. Mesmo sabendo desta heterogeneidade entre os estudantes recebidos por um professor, sua intenção é levá-los para o ponto de chegada mais próximo do que ele considera ideal, aquele que corresponde ao objetivo de aprendizagem determinado pelo docente para aquela unidade de estudo. Cada indivíduo poderá, sim, fazer trajetos diferentes e particulares, mas quanto mais se aproximarem os pontos de chegada, maior terá sido o sucesso da atividade docente, tal como mostra a Figura 2. 
FIGURA 02 - Trajetórias onde os pontos de partida são distintos e os pontos de chegada próximos ao planejado.

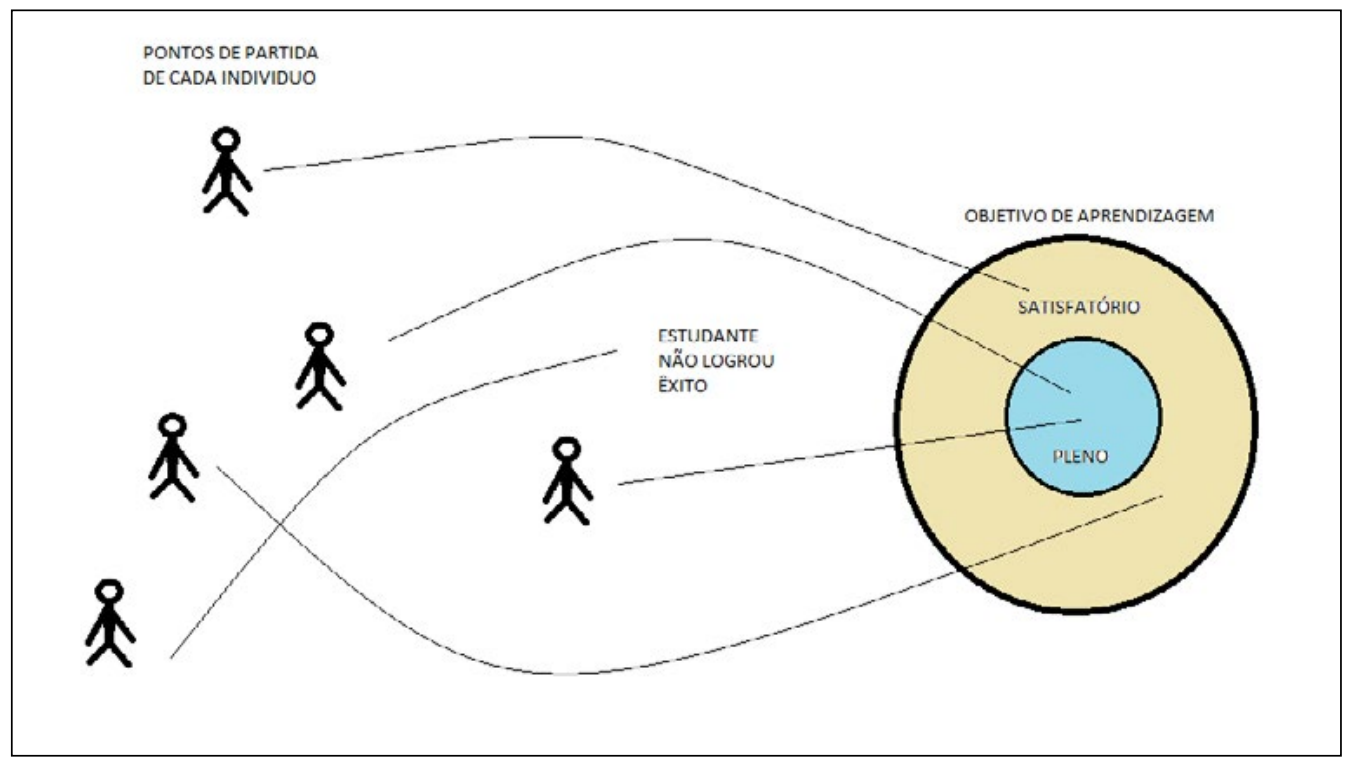

Fonte: dos autores

Neste contexto, o presente estudo buscou entender se a aplicação da metodologia Peer Instruction fornece suporte aos educandos para a construção de trajetórias de aprendizagem onde os diferentes pontos de chegada, depois de um semestre de aulas, são mais próximos uns dos outros. Em outras palavras, o objetivo é saber se o desempenho coletivo médio é melhor (média maior e desvio-padrão menor) quando se aplicam metodologias ativas (neste caso o Peer Instruction), comparando as médias finais dos estudantes submetidos a essa metodologia em relação aos demais.

\section{TRABALHOS RELACIONADOS}

Vários estudos investigam as dificuldades de ensino e aprendizagem de algoritmos, propondo alternativas metodológicas ou instrumentais para auxiliar neste processo como, por exemplo, os realizados por Falckembach e De Araujo (2013), Barcelos (2013), Noschang; Pelz e Raabe (2014), Ferreira e Reategui (2016), Valdez et al. (2017), Bulcão, Campos Neto e Moreira (2017), Giraffa e Müller (2017), Barros e Santos (2018), Schorr, Gomes e Pretto (2018).

Um sistema de tutoria web para apoiar o aprendizado de programação Java é apresentado por Cabada et at. (2015). O ambiente denominado de Intelligent Learning Environment (ILE) usa diferentes metodologias de aprendizagem para apoiar o estudante na solução de problemas de programação. Nitin, Rao e Sivaraman (2011) fazem um estudo sobre aspectos 
emocionais envolvidos no aprendizado e apresentam o desenvolvimento de um modelo de reconhecimento de emoções em sistemas de aprendizado online com o intuito de tornar o aprendizado de algoritmos mais efetivo. Alepis et al. (2018) apresenta uma ferramenta para ensinar princípios básicos de programação de computadores aos estudantes do ensino fundamental. A proposta da ferramenta é ensinar lógica de programação apresentando a teoria de forma lúdica.

\section{METODOLOGIA}

Para levar a cabo o objetivo deste trabalho, realizou-se um estudo tomando como referência as notas de desempenho (as médias finais) dos estudantes da disciplina Algoritmos e Programação em turmas onde se aplicou metodologias convencionais em comparação com alunos onde a metodologia Peer Instruction foi utilizada. A disciplina está presente em todos os currículos dos cursos de Tecnologia da Informação (TI) da <universidade omitida para avaliação $>$, locus do estudo. O desempenho médio dos estudantes em condições normais foi considerado desde 2009, quando houve a implantação da atual ementa, e comparado ao desempenho médio alcançado por quatro turmas onde houve a aplicação do Peer Instruction no primeiro semestre de 2016, 2017, 2018 e 2019. O grupo de controle, então, é formado por 421 estudantes de diferentes turmas da disciplina e o grupo experimental é composto por 59 estudantes. Os estudantes que abandonaram a disciplina, ou seja, não realizaram todas as avaliações, foram desconsiderados para não distorcer as médias e manter as duas bases nos mesmos patamares de comparabilidade.

A pesquisa é de natureza aplicada, uma vez que é dirigida para um problema específico, ou seja, a influência da metodologia Peer Instruction no desempenho dos estudantes de Algoritmos e Programação. Quanto aos objetivos, caracteriza-se como uma pesquisa exploratória, pois tem como intenção proporcionar maior familiaridade com o problema, com vistas a tornálo mais explícito ou a construir novas hipóteses (GIL, 2008). A técnica e método procedimental é quasi-experimental, uma vez que a pesquisa possui as mesmas características de pesquisa experimental, contendo um objeto de estudo e um conjunto de variáveis que se pretende analisar e observar de forma controlada, porém o campo de estudos é limitado a um universo restrito, ou seja, a uma amostra escolhida por conveniência, não podendo se obter conclusões generalizáveis, mas conclusões que se aplicam aos dados estudados e que, por analogia, podem se repetir em outra amostra (CAMPBELL; STANLEY, 2015). Os dados coletados foram abordados de forma qualitativa com apoio de ferramentas quantitativas de análise estatística.

A metodologia Peer Instruction foi utilizada no grupo experimental para consolidar a compreensão teórica dos diferentes pontos do conteúdo programático ao longo do semestre. As intervenções ocorreram ao iniciar a abordagem dos conteúdos: a) comandos de entrada e saída; b) expressões 
aritméticas, lógicas e operadores relacionais; c) desvio condicional; d) expressões literais (strings); e) estruturas de repetição; f) vetores e matrizes e g) modularização.

A Figura 03 apresenta um dos testes conceituas aplicados, neste caso após a abordagem do tópico desvio condicional ${ }^{8}$. Inicialmente os estudantes foram convidados a analisar o programa e marcar a alternativa (resposta) que julgavam ser mais adequada à interpretação que eles haviam feito do código. Em um segundo momento os alunos buscam um ou dois colegas (formando grupos de dois ou três membros) que pensaram de forma diferente e não responderam ao questionamento da mesma forma, para discutir, debater, argumentar, contra-argumentar e obter uma resposta consensual.

A título de exemplo, a Figura 04 apresenta o resultado de um dos testes de conhecimento (da turma de 2018), feito com o questionamento apresentado na Figura 03 , mostrando que houve um incremento no índice de acertos na ordem de $134 \%$, passando de $39,1 \%$ de respostas certas para $91,7 \%$, entre o teste inicial e o teste realizado depois da discussão em grupo. Ressalta-se que a reflexão final que o professor realiza, comentando o resultado e as razões pela qual as demais respostas estão erradas, corrobora para consolidar o aprendizado e permitir que as dúvidas ainda persistentes sejam sanadas, especialmente pelos estudantes que não foram convencidos, durante a discussão em grupo, pelas explicações e argumentações dos colegas.

8 A importância das chaves para delimitar os blocos de código que estarão condicionados a expressão lógica da instrução "if" é parte integrante do conteúdo deste tópico. 
FIGURA 03 - Exemplo de instrumento usado para obtenção de feedback

\section{Desvio condicional}

public class Exercicio

\{

public static void main(String[ args)

\{

int $x=$ Entrada.leialnt();

if $(x>10)$

\{

System.out.println("O número é positivo e maior do que 10");

if $(x>20)$

\{

System.out.println("e tambèm é maior do que $20 "$ );

else

\}

\{

\}

System.out.println("O número é positivo e menor ou igual do que $10 "$ );

\}

Considere que o usuário deste programa vai entrar com o valor 15. Qual será o resultado produzido pelo programa?*

O programa vai exibir a frase "O número é positivo e maior do que 10 ".

0 programa vai exibir duas frases. A primeira "O número é positivo e maior

$\bigcirc$ do que 10" e depois vai exibir "O número é positivo e menor ou igual do que $10 "$.

O programa não vai exibir nada, simplesmente porque há um erro que impede sua compilação e portanto o sistema não roda.

Fonte: dos autores 
FIGURA 04 - Exemplo índice de acertos dos questionamentos

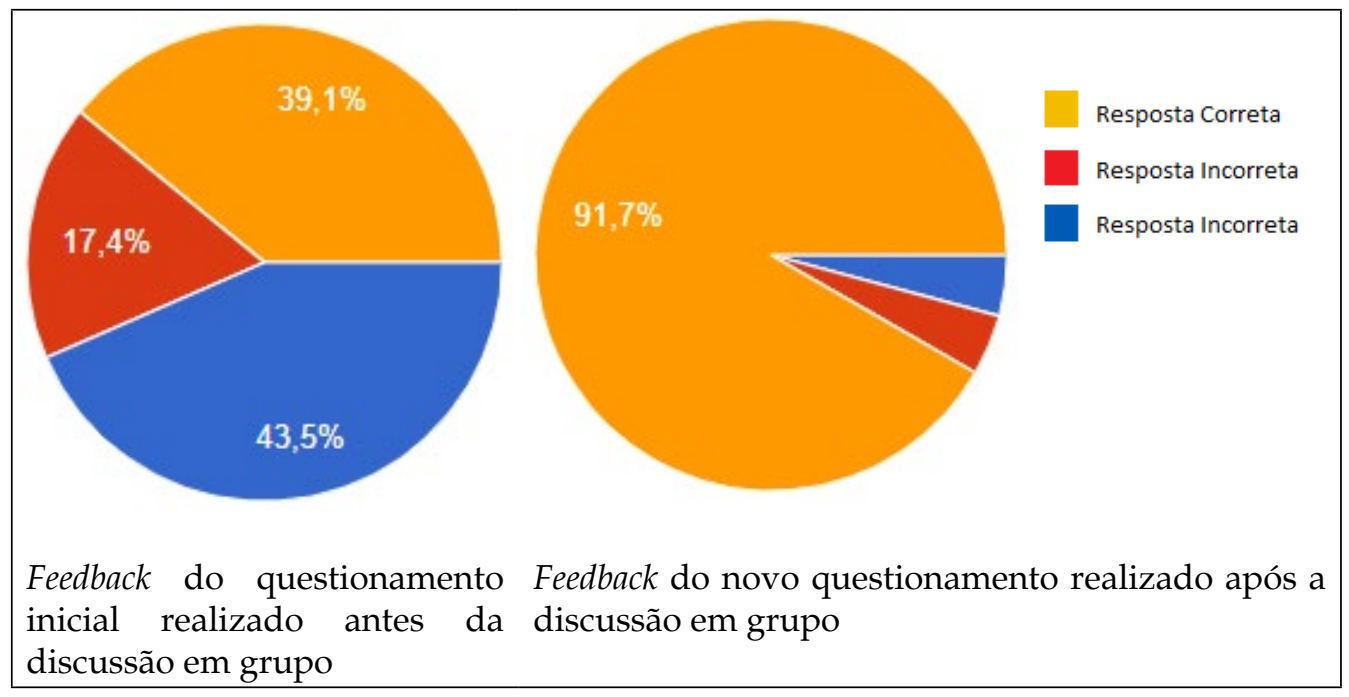

Fonte: dos autores (2019)

\section{RESULTADOS E DISCUSSÃO}

Com auxílio do software de análise estatística SPSS (Statistical Package for the Social Sciences), foram obtidas as médias de desempenho dos estudantes dos dois grupos, ou seja, os alunos que foram expostos a metodologia ativa Peer Instruction e os que obtiveram aulas convencionais. O Quadro 01 apresenta os resultados calculados.

QUADRO 01 - Resultados estatísticos descritivos

Estatisticas descritivas (SPSS) do grupo submetido à metodologia Peer Instrusction

\begin{tabular}{|l|r|r|r|r|r|r|}
\hline & \multicolumn{1}{|c|}{$\mathrm{N}$} & \multicolumn{1}{c|}{ Intervalo } & \multicolumn{1}{c|}{ Mínimo } & \multicolumn{1}{c|}{ Máximo } & Média & Desvio Padrão \\
\cline { 2 - 7 } & Estatística & Estatística & Estatística & Estatística & Estatística & Estatística \\
\hline Nota & 59 & 55 & 45 & 100 & 87,05 & 12,933 \\
N válido (de lista) & 59 & & & & & \\
\hline
\end{tabular}

Estatísticas descritivas (SPSS) do grupo não submetido à metodologia Peer losstrustion.

\begin{tabular}{|l|r|r|r|r|r|r|}
\hline & \multicolumn{1}{|c|}{$\mathrm{N}$} & Intervalo & Mínimo & Máximo & Média & Desvio Padrão \\
\cline { 2 - 7 } & Estatística & Estatística & Estatística & Estatística & Estatística & Estatística \\
\hline Nota & 421 & 100 & 0 & 100 & 81,19 & 18,069 \\
N válido (de lista) & 421 & & & & & \\
\hline
\end{tabular}

Fonte: elaborado pelos autores com uso do SPSS (2019) 
Observa-se que a média final dos estudantes do grupo experimental foi 8,7 com desvio padrão próximo de 1,3 , enquanto a média dos demais estudantes foi 0,6 pontos menores com escore 8,1 e desvio padrão maior, em torno de 1,8 . Os dados indicam, em uma primeira análise, uma resposta positiva ao questionamento inicial, mostrando que a aplicação da metodologia corroborou para melhoria do desempenho e aproximação dos resultados finais, uma vez que o desvio-padrão do grupo experimental foi 0,5 pontos menores do que o desvio-padrão da média dos demais estudantes.

Para averiguar, no entanto, se esse resultado é significativo, ou seja, saber se a média dos estudantes do grupo experimental é efetivamente superior à média dos demais alunos, os dados foram submetidos a um teste de hipótese. O teste de normalidade Kolmogorov-Smirnov indicou que os dados não possuem distribuição estatística normal, portanto, inadequados para que sejam submetidos a um teste de hipótese paramétrico (teste $\mathrm{Z}$ ou teste t-Student, por exemplo). Aplicou-se, então, o teste Mann-Whitney que é um teste não paramétrico utilizado para comparar a distribuição dos valores de duas amostras de dados independentes.

QUADRO 02 - Resultado do teste de hipótese

\begin{tabular}{|c|c|c|c|c|}
\hline & Hipótese nula & Teste & Sig. & Decisão \\
\hline 1 & $\begin{array}{l}\text { A distribuição de Nota é a mesma } \\
\text { entre as categorias de Metodologi }\end{array}$ & $\begin{array}{l}\text { Teste U de } \\
\text { Mann- } \\
\text { Whitney de } \\
\text { iamostras } \\
\text { independente } \\
\text { s }\end{array}$ & .017 & $\begin{array}{l}\text { Rejeitar a } \\
\text { hipótese } \\
\text { nula. }\end{array}$ \\
\hline
\end{tabular}

São exibidas significâncias assintótic as. O nivel de significância é ,05.

Fonte: elaborado pelos autores com uso do SPSS (2019)

O Quadro 02 apresenta o resultado do teste de hipótese, indicando que as amostras são significativamente diferentes. $\mathrm{O}$ teste de hipótese procura confirmar que as amostras não têm diferenças estatísticas, ou seja, de que não há diferenças importantes entre as notas dos estudantes do grupo experimental e controle. Como o nível de significância dessa hipótese ficou abaixo de 5\% (apenas 1,7\%), a hipótese foi rejeitada, indicando amostras diferentes. Isso permite afirmar que a distribuição das notas de desempenho, obtidas pelos estudantes expostos a metodologia Peer Instruction, difere das demais notas.

Ao comparar as médias finais do grupo experimental (Quadro 01) com os demais estudantes é possível observar que os alunos expostos a metodologia têm notas finais superiores e mais homogêneas. $\mathrm{O}$ teste de hipóteses corrobora para confirmar que essa metodologia pode contribuir positivamente para a melhoria do aprendizado de algoritmos, reforçando o entendimento de que as metodologias ativas são importantes aliadas do processo de construção de 
conhecimento. Esse resultado é reforçado se consideramos as médias de cada uma das turmas separadamente. Os 421 alunos do grupo de controle estavam distribuídos em 40 turmas e em $70 \%$ dos casos (28 turmas) a média geral daquela turma foi inferior à média geral do grupo experimental.

\title{
5. CONSIDERAÇÕES FINAIS
}

A intenção docente é levar a maioria dos estudantes para o ponto de chegada mais próximo do objetivo pedagógico planejado (Figura 01). Mas cada estudante tem esquemas mentais de raciocínio diferente, conhecimentos prévios distintos e, portanto, percorre sua própria trajetória. Neste cenário, o presente estudo reforçou a importância das metodologias ativas para o processo de aprendizagem, aproximando os estudantes do ponto de chegada.

O uso da metodologia Peer Instruction, como continuidade deste trabalho, pode ser ampliado para outras turmas de disciplinas subsequentes, avaliando a progressão dos estudantes no curso. Uma outra questão que também merece mais atenção e investigação diz respeito aos estudantes que não se engajam ou não se dispõe a aprender de forma ativa. Empiricamente foi possível observar que os estudantes menos participativos e menos colaborativos foram também aqueles com menor desempenho. Propor estratégias para aumentar o engajamento dentro do contexto de aplicação dessa metodologia ativa pode ser um tema de investigação futura, pois ficou perceptível uma maior receptividade para a metodologia Peer Instruction nas primeiras intervenções, ou seja, a empolgação com a novidade e a aceitabilidade da metodologia reduziu à medida que o método era repetido.

\section{REFERÊNCIAS}

ABU-ODA, Ghadeer S.; EL-HALEES, Alaa M. Data mining in higher education: university student dropout case study. In: International Journal of Data Mining \& Knowledge Management Process, v. 5, n. 1, 2015.

\author{
ALEPIS, E.; TROUSSAS, C. M-learning programming platform: Evaluation in \\ elementary schools. Informatica, n. 41 (4), pp. 471-478, 2017.
}

\begin{abstract}
ALVES, Alessandro Caldeira; GUIMARÃES, Larissa Moreira; PIMENTA, Thais Silveira. A utilização de Tecnologias de Informação e Comunicação (TICs) como ferramenta para minimizar os altos índices de retenção e evasão na disciplina de Função de uma variável (Cálculo I) no BCT/UFVJM. In: Simpósio Tecnologias e Educação a Distância no Ensino Superior, v. 1, n. 1, 2018.
\end{abstract}

BARBOSA, Eduardo Fernandes; DE MOURA, Dácio Guimarães. Metodologias ativas de aprendizagem na educação profissional e tecnológica. Boletim Técnico do Senac, v. 39, n. 2, p. 48-67, 2013. Disponível em: <http:/ /www.bts.senac.br/index.php/bts/ article/view/349/333> 
BARCELOS, Ricardo José dos Santos. O processo de construção do conhecimento de algoritmos com o uso de dispositivos móveis considerando estilos preferenciais de aprendizagem. Porto Alegre, 2013.

BARCELOS, Thiago Schumacher; SILVEIRA, Ismar Frango. Pensamento computacional e educação matemática: Relações para o ensino de computação na educação básica. In: XX Workshop sobre Educação em Computação, Curitiba. Anais do XXXII CSBC. 2012. p. 23.

BARROS, Djalmira de Sá Almeida; SANTOS, J. R. A. Técnicas de estudo e gestão do tempo no auxílio a aprendizagem de fundamentos de algoritmos e lógica aplicada a computação. CIMATech, v. 1, n. 5, 2018.

BERBEL, Neusi Aparecida Navas. As metodologias ativas e a promoção da autonomia de estudantes. Semina: Ciências Sociais e Humanas, v. 32, n. 1, p. 25-40, 2011.

BERNHEIM, Carlos. Chaui. In: Desafios da Universidade na sociedade do conhecimento: cinco anos depois da Conferência Mundial sobre Educação Superior. Brasília. 2008. Disponível em: <https:/ / unesdoc.unesco.org/ark:/48223/ pf0000134422_por>

BONDIOLI, Ana Cristina Cristina Vigliar; VIANNA, Simone Cristina Gonçalves; SALGADO, Maria Helena Veloso. Metodologias ativas de Aprendizagem no Ensino de Ciências: práticas pedagógicas e autonomia discente. Caleidoscópio, v. 2, n. 10, p. 23-26, 2019.

BRASIL. Ministério da Educação. Diretrizes Curriculares Nacionais para os cursos de Computação. Brasilia: MEC, 2016. Disponível em: <http:/ / portal.mec.gov.br/ component/content/article?id=12991>

BRASIL. Instituto Nacional de Estudos e Pesquisas Educacionais. Sinopses Estatísticas da Educação Superior 2017. Brasilia: Inep, 2018. Disponível em: <http:/ / portal.inep.gov.br/web/guest/sinopses-estatisticas-da-educacao-superior>

BULCÃO, J. D. S. B.; CAMPOS NETO, E. B.; MOREIRA, K. C. Mapping on the teaching of algorithms and computational logic in the degree courses of computer science and computing in higher education institutions in Brazil. In: CEUR Workshop Periódico científico acadêmicos, Ctrl+E 2017 - Anais do II Congresso sobre Tecnologias na Educacao, Periódico científico acadêmicos of the 2nd Congress on Technology in Education, Ctrl + E 2017. Natal, 2017.

CABADA, R. Z. et al. An affective learning environment for Java. In: Proceedings of IEEE 15th International Conference on Advanced Learning Technologies: Advanced Technologies for Supporting Open Access to Formal and Informal Learning, pp. 350354. 2015.

CAMPBELL, Donald T.; STANLEY, Julian C. Experimental and quasi-experimental designs for research. Ravenio Books, 2015. 
CASTANHA, Débora; DE CASTRO, Maria Bernadete. A necessidade de refletir sobre as estratégias pedagógicas para atender à aprendizagem da geração Y. Revista de EDUCAÇÃO do Cogeime, v. 19, n. 36, p. 27-38, 2010. Disponível em: <https:/ /www. redemetodista.edu.br/revistas/revistas-cogeime/index.php/COGEIME/article/ view $/ 70 / 70>$

FALCKEMBACH, Gilse A. Morgental; DE ARAUJO, Fabrício Viero. Aprendizagem de algoritmos: dificuldades na resolução de problemas. In: Anais Sulcomp, v. 2, 2013.

FERREIRA, Vinicius Hartmann; REATEGUI, Eliseo Berni. Uma Comunidade de Prática para o ensino e a aprendizagem de programação. Revista novas tecnologias na educação. Porto Alegre, RS, vol. 14, n. 2, p.1-11, dez 2016.

FREEMAN, Scott et al. Active learning increases student performance in science, engineering, and mathematics. In: Proceedings of the National Academy of Sciences, v. 111, n. 23, p. 8410-8415, 2014.

GIL, Antonio Carlos. Métodos e técnicas de pesquisa social. 6. ed. São Paulo: Editora Atlas, 2008.

GIRAFFA, Lucia Maria Martins; MÜLLER, Luana. Metodologia baseada em sala de Aula invertida e Resolução de Problemas relacionado ao cotidiano dos estudantes. Brazil, South America, 2017.

HOED, Raphael Magalhães. Análise da evasão em cursos superiores: o caso da evasão em cursos superiores da área de Computação (Dissertação de Mestrado). Universidade de Brasília. Brasilia, 2016.

MAZUR, Eric; HILBORN, Robert C. Peer instruction: A user's manual. Physics Today, v. 50, n. 4, p. 65, 1997.

MITRE, Sandra Minardi et al. Metodologias ativas de ensino-aprendizagem na formação profissional em saúde: debates atuais. Ciência \& saúde coletiva, v. 13, p. 2133-2144, 2008. Disponível em: <https:/ / www.scielosp.org/scielo.php?pid=S1413$81232008000900018 \&$ script=sci_arttext\&tlng=es>

NOSCHANG, Luis Fernando; PELZ, Fillipi; RAABE, A. Portugol studio: Uma ide para iniciantes em programaçao. In: Anais do CSBC/WEI, pp. 535-545, 2014.

NITIN, K. L.; RAO, L. L. N.; SIVARAMAN, N. K. An emotional system for effective and collaborative e-learning. In: Proceeding of 4th International Conference on Advances in Computer-Human Interactions, pp. 260-266. 2011.

RAABE, André Luís Alice; SILVA, J. M. C. Da. Um ambiente para atendimento as dificuldades de aprendizagem de algoritmos. In: XIII Workshop de Educação em Computação. São Leopoldo, RS, Brasil, 2005. 
SCHORR, Maria; GOMES, Eduardo Rodrigues; PRETTO, Fabrício. Aprendizagem de Algoritmos e Programação por meio da ferramenta visual HelpBlock. In: Anais do Workshop do Congresso Brasileiro de Informática na Educação, 2018.

TORRES, Patrícia Lupion. Laboratório on line de aprendizagem: uma proposta crítica de aprendizagem colaborativa para a educação (Tese de Doutorado). Universidade Federal de Santa Catarina, Florianópolis, 2002.

VALDEZ, Mario García et al. Enhancing Student Engagement via Reduction of Frustration with Programming Assignments using Machine Learning. In: Proceedings of International Joint Conference on Computational Intelligence, pp. 297-304, 2017.

VASCONCELOS, Andreza Cavalcanti et al. As estratégias de ensino por meio das metodologias ativas/Teaching strategies through active methodologies. Brazilian Journal of Development, v. 5, n. 5, p. 3945-3952, 2019.

VIEIRA, Carlos Eduardo Costa; DE LIMA JUNIOR, José Augusto Teixeira; DE PAULA VIEIRA, Priscila. Dificuldades no Processo de Aprendizagem de Algoritmos: uma Análise dos Resultados na Disciplina de AL1 do Curso de Sistemas de Informação da FAETERJ-Campus Paracambi. Cadernos UniFOA, v. 10, n. 27, p. 5-15, 2015. 